\title{
Microvascular effects of microneedle application
}

Johannes Hackethal, Fredrik Iredahl, Joakim Henricson, Chris Anderson and Erik Tesselaar

The self-archived postprint version of this journal article is available at Linköping University Institutional Repository (DiVA):

http://urn.kb.se/resolve?urn=urn:nbn:se:liu:diva-168535

N.B.: When citing this work, cite the original publication.

Hackethal, J., Iredahl, F., Henricson, J., Anderson, C., Tesselaar, E., (), Microvascular effects of microneedle application, Skin research and technology. https://doi.org/10.1111/srt.12918

Original publication available at:

https://doi.org/10.1111/srt.12918

Copyright: John Wiley and Sons

http://www.wiley.com/ 


\section{Microvascular effects of microneedles application}

Johannes Hackethal ${ }^{1,2}$, Fredrik Iredah| ${ }^{3,4}$, Joakim Henricson ${ }^{5,6}$, Chris D. Anderson ${ }^{6,7}$, Erik Tesselaar ${ }^{9}$

1 Ludwig Boltzmann Institute for Experimental and Clinical Traumatology, Vienna, Austria

2 Austrian Cluster for Tissue Regeneration, Vienna, Austria

3 Department of Health, Medicine and Caring Sciences, Linköping University, Division of Community Medicine, Linköping, Sweden

4 Department of Primary health care, Region Östergötland, Linköping, Sweden

5 Department of Emergency Medicine, Local Health Care Services in Central Östergötland, Region Östergötland, Linköping, Sweden

6 Department of Biomedical and Clinical Sciences, Division of Cell Biology, Linköping University, Linköping, Sweden

7 Department of Dermatology and Venerology, Heart and Medicine Centre, Region Östergötland, Linköping, Sweden

8 Department of Radiation Physics and Department of Clinical and Experimental Medicine, Linköping University, Linköping, Sweden.

\section{Corresponding author:}

Johannes Hackethal, PhD

Adress: Donaueschingenstrasse 13, 1200 Vienna, Austria

Mail: Johannes.Hackethal@trauma.lbg.ac.at

Telephone: +43(0) 593 93-41248

This work was performed at the Department of Hand and Plastic surgery, Burns and Intensive care and Department of Clinical and Experimental Medicine, Linköping University, 58185 Linköping, Sweden.

Running head: Effects of microneedles on skin microcirculation 


\section{Abstract}

\section{Background}

The efficiency of transdermal drug delivery may be increased by pretreating the skin with microneedles, but distinct effects of microneedles and the microneedle-enhanced delivery of vasoactive drugs on the skin microvasculature are still not well investigated.

\section{Materials and Methods}

In eight healthy human subjects, we measured the microvascular response to microneedle-induced microtraumas in the skin microvasculature using polarized light spectroscopy imaging (Tissue Viability imaging, TiVi). The microvascular response was assessed for up to 48 hours for three microneedle sizes (300 $\mu \mathrm{m}, 500 \mu \mathrm{m}$, and $750 \mu \mathrm{m})$ and for different pressures and application times.

\section{Results}

In our results, microneedle application increased the local red blood cell (RBC) concentration for up to 24 hours dependent on the needle lengths, applied time and force.

\section{Conclusion}

Optimization of microneedles size, pressure and application time should be taken into account when future protocols for drug delivery as well as experimental provocations.

Keywords: microneedles, TiVi imaging, skin provocation 


\section{Introduction}

The human skin forms an important biological barrier that protects against external bacterial access and everyday mechanical damage, meanwhile preventing internal loss of water and other molecules. In the 1970's, the microneedle technique was introduced to enhance transdermal drug delivery (TDD). When pressed onto the human skin, microneedles penetrate the epidermis and parts of the dermis and form microchannels. During the past decades, TDD has become a popular technique to apply drugs over the skin barrier, ${ }^{1,2}$ because it is an easy-to-perform method to deliver drugs dermally - neither passing the highly enzymatically active gastrointestinal tract nor undergoing the first pass effect in the liver. ${ }^{3-5}$ TDD is frequently used for vaccination studies or diagnosis because conventional stainless-steel needles may be associated with fear and discomfort, especially among children. ${ }^{6}$ In addition, the needed drug doses are significantly lower compared to oral medication and conventional needles, making TDD applications cheaper. ${ }^{7}$

In general, microneedles can be divided into three main categories: solid, degradable and hollow. Solid microneedles can be metal or polymer made. Stainless steel microneedles have sufficient mechanical strength, are easy to form and FDA approved. Degradable microneedles consist of polymers, are inexpensive, biocompatible and in case of breakage they will be eliminated by the body. 3,8

Currently used microneedles typically vary in length between $50-900 \mu \mathrm{m} .^{3,8}$ However, for effective piercing of the epidermis, the minimally required needle length is generally considered to be at least $300 \mu \mathrm{m} .{ }^{9}$ An increase in needle numbers does not increase pain where as an increase in needle length above $900 \mu \mathrm{m}$ may significantly increase pain, because the needles may stimulate the local sensory nerves. ${ }^{10,11}$ Immune and micro trauma responses in the skin induce both transepidermal water loss and erythema, ${ }^{9}$ which correlates to the inflammatory response of the damaged microtissue and the applied drugs. ${ }^{12}$

By forming micropores through the epidermis, a therapeutic window can be produced, as the local microtraumas start to induce a transdermal response (e.g. by keratinocytes, $95 \%$ of all cells in the epidermis). These cells activate a cascade of inflammatory response including cytokines (mainly II-1 $\alpha$, but also IL-6, IL-8, granulocyte-macrophage colony stimulatory factor and TNF- $\alpha$ ). A reactive vasodilatation, which increases the red blood cell (RBC) concentration, enables a cell infiltration towards the epidermis to initiate the final restoration of the skin barrier function. ${ }^{9,12}$

A lot of useful applications of microneedles have already been demonstrated, including the use of microneedles as delivery systems for vaccines, ${ }^{6,7}$ as skin allergy test devices, ${ }^{13,14}$ for stimulating 
angiogenesis by electrical signals and growth factors, for applications of immunobiologicals, ${ }^{4}$ biopharmaceuticals, ${ }^{15}$ drugs, ${ }^{5}$ and for diagnosis purposes, ${ }^{16}$ or as cosmetic products. ${ }^{3}$ However, the direct and prolonged effects of microneedle application on the local microcirculation have not been studied extensively.

The aim of this study was to objectively measure microvascular effects of microneedles in the skin by different needle lengths, durations and weights using polarized light spectroscopy imaging.

\section{Material and Methods}

\section{Subjects}

Eight healthy volunteers (4 male), 21-37 years (mean \pm SD, $24.9 \pm 6.1$ years) and Body Mass Index (BMI) 19-26.3 (mean $\pm S D, 22 \pm 2.8$ ) were recruited. The subjects had no known skin or vascular disease or use of medications (except oral contraceptives) and gave their informed consent to participate. Blood pressures were measured before the experiments (mean $\pm S D$, systolic $115 \pm 8.5$; diastolic $71 \pm 6.8$ ). The participants were asked to refrain from drinking caffeine or tea, use nicotine products and eating for two hours prior to the experiments. All experiments were approved by the regional ethics review board at Linköping University, Sweden.

\section{Study protocol}

All measurements were performed in a temperature-controlled room at $21 \pm 1^{\circ} \mathrm{C}$. All participants were in a semi-supine position in a bed for at least 10 minutes before the measurements started. All measurements were done on the forearms which were disinfected with a skin cleaner (Klorhexidin 5 $\mathrm{mg} / \mathrm{ml}$, Fresenius Kabi AB, Uppsala, Sweden) before the experiments. Stainless steel microneedles arrays were used in all experiments ( 2 rows à 8 microneedles, $300 \mu \mathrm{m}, 500 \mu \mathrm{m}$ and $750 \mu \mathrm{m}$ lengths (Dermaroller GmbH, Wolfenbuettel, Germany).

Microneedles with a length of $300 \mu \mathrm{m}, 500 \mu \mathrm{m}$ and $750 \mu \mathrm{m}$ were gently applied on the forearms using a weight of $100 \mathrm{~g}$ or $500 \mathrm{~g}$ for 10 or 60 seconds. TiVi images were acquired every 10 seconds in the first 5 minutes and every 60 seconds for the next 55 minutes. Additional images were acquired after 6,12 , 24 and 48 hours.

\section{Equipment}


The microvascular response was quantified by Tissue Viability imaging (TiVi). ${ }^{17}$ Briefly, TiVi is based on a digital camera and two filters (polarizers). Outgoing light from the camera flash passes the first filter producing linear-polarized light. A part of this light will penetrate the skin and become randomly scattered (depolarized). Part of the backscattered, randomly polarized light will pass the second filter placed in front of the camera lens. RBCs absorb light in a range of $500-600 \mathrm{~nm}$ (the green wavelength region) to a much higher extent than light in a range of $600-700 \mathrm{~nm}$ (the red wavelength region), in comparison to the surrounding tissue, which absorbs red and green light at approximately the same amount. ${ }^{18}$ These differences in absorption can be determined and calculated into a measure of RBC concentration $\left(C_{R B C}\right)$. The polarized digital TiVi camera was placed $20-30 \mathrm{~cm}$ above the skin. Ambient light was turned off during all measurements. The images were reduced in size and cropped into the area of interest using image processing software (ImageJ, US National Institutes of Health, Maryland, USA) and converted into numerical data using the software provided by the TiVi system (TiVi 7001.1 WheelsBridge AB, Linköping, Sweden).

\section{Data analysis}

Data is presented as mean \pm SD or as mean \pm SEM, if not stated otherwise. Statistical analysis was further performed using a 2-tailed (no priori assumption), paired Student's t-test (comparing means from the same group of subjects, before and after treatment). The microneedle time series tests were further analyzed using 2-way ANOVA with multiple comparisons. $\mathrm{P}<0.05$ was considered as statistically significant. All statistical analysis was calculated using GraphPad Prism version 6.00 for Windows (GraphPad Software, San Diego California USA, “www.graphpad.com”).

\section{Results}

During the first $60 \mathrm{~min}$ after application of microneedles to the skin, a significant increase in $C_{R B C}$ was observed for all needle lengths, application weights and durations (Figure 1 - A). This increase in $C_{R B C}$ was visible as local erythema, at the sites where the needles penetrated the epidermis. Mean $C_{R B C}$ increased with the length of the needles, independent of duration or weight (Table 1). Significant differences in $C_{R B C}$ were observed between $300 \mu \mathrm{m}$ and $750 \mu \mathrm{m}$, but no other groups. Microneedle application of $500 \mathrm{~g}$ and $10 \mathrm{~s}$ duration increased $C_{R B C}$ more than the needle application using $100 \mathrm{~g}$ weight and $60 \mathrm{~s}$ duration. Microneedle application of $500 \mathrm{~g}$ and $60 \mathrm{~s}$ showed the highest absolute $\mathrm{C}_{\mathrm{RBC}}$ increase, still visible after 60 minutes, and therefore, a time series up to 48 hours was performed (Figure $1-B$ and $C)$. A significant increase in $C_{R B C}$ after $24 \mathrm{~h}$ was observed with the longest needles $(750 \mu \mathrm{m})$ 
applied with $500 \mathrm{~g}$ for $60 \mathrm{~s}(\mathrm{p}=0.047)$. Shorter needles and less weight and duration resulted in a faster return to the baseline of the $C_{R B C}$ (Table 2). After 48 hours, no significant increase in $C_{R B C}$ was observed in any of the experiments.C

\section{Discussion}

The main finding of this study is that the application of microneedles to skin of the forearm results in immediate vasodilatation and this vasodilatory response depends on the needle length, application weight and duration. We observed a substantial variation in maximum response between subjects. This variability in microvascular response may be caused by individual variations in vascular reactivity and circadian variations due to different measuring time points (between 7 a.m. and 9 p.m). ${ }^{17}$

All participants described the microneedle applications as painless, which is consistent with previous findings. ${ }^{9,4}$

This experiment showed that longer needles increased the $C_{R B C}$ response, which was also described by other studies, ${ }^{11,19}$ due to the fact that more microvascular tissue is damaged, leading to an elevated immunological responses by keratinocytes, ${ }^{9}$ Langerhans cells and an accumulation of red blood cells. ${ }^{17}$ This study also showed that an increase of applied mass on the skin can elevate the concentration of RBC more than an increase of application time: $500 \mathrm{~g}$ weight for only $10 \mathrm{~s}$ application time showed higher $C_{R B C}$ responses when compared to $100 \mathrm{~g}$ weight for $60 \mathrm{~s}$ application time. Similar results were also shown by Noh et al., where microneedle applications showed the same extent of skin redness after application on the forearm, as measured using reflectance spectrophotometry, regardless if applied for 2 or 240 min. 20

In the 48-hour tests, $300 \mu \mathrm{m}$ needle lengths provoked an increase in $C_{R B C}$ up to 6 hours when applied for $60 \mathrm{~s}$ and $500 \mathrm{~g}$, but when using lower mass or duration, erythema was not detectable any more after 6 hours. A significantly increased $C_{R B C}$ concentration was observed up to 24 hours for the longest needles applied with $500 \mathrm{~g}$ for 60 minutes.

Bal et al., ${ }^{9}$ Haq et al. ${ }^{21}$ and Han et al. ${ }^{12}$ showed that the formation of erythema recovered after $48 \mathrm{~h}$ latest for microneedle sizes shorter than $300 \mu \mathrm{m}$, but they used different microneedle shapes. Since needle shape is known to be an important parameter in barrier disruption ${ }^{9}$, the results of these studies cannot easily be compared to the findings in this study. Also, in contrast with our study, the previous 
studies used laser Doppler flowmetry or a reflectance spectrometry to detect skin responses, which may also have different detection properties than TiVi imaging.

\section{Conclusion}

The results of the current study show that the microvascular skin reactions caused by microneedle application can be measured reliably and practically using polarized light spectroscopy (TiVi). The findings confirm previous findings that the extent of the microvascular response is dependent on both the needle length and the weight at which the needle arrays are applied.

From a clinical point of view, microneedles of $750 \mu \mathrm{m}$ length, although painless during application, cause an elevated red blood cell concentration for up to 24 hours.

\section{Conflict of interest}

The authors declare on conflicts of interest.

\section{References}

1. Akhtar, N. Microneedles : An Innovative approach to Transdermal Delivery - A Review. Int. J. Pharm. Pharm. Sci. 6, 18-25 (2014).

2. Dragicevic, N. \& Maibach, H. Combined use of nanocarriers and physical methods for percutaneous penetration enhancement. Adv. Drug Deliv. Rev. 127, 58-84 (2018).

3. Bariya, S. H., Gohel, M. C., Mehta, T. a. \& Sharma, O. P. Microneedles: An emerging transdermal drug delivery system. J. Pharm. Pharmacol. 64, 11-29 (2012).

4. Cleary, G. W. Microneedles for drug delivery. Pharm. Res. 28, 1-6 (2011).

5. Wang, Y., Thakur, R., Fan, Q. \& Michniak, B. Transdermal iontophoresis: Combination strategies to improve transdermal iontophoretic drug delivery. Eur. J. Pharm. Biopharm. 60, 179-191 (2005).

6. Shin, C. I., Seong, D. jeong, Rejinold, N. S. \& Kim, Y.-C. Microneedles for vaccine delivery: challenges and future perspectives. Ther. Deliv. 8, 447-460 (2017).

7. Cary, J. H., Li, B. S. \& Maibach, H. I. Dermatotoxicology of microneedles (MNs) in man. Biomed. Microdevices 21, 1-8 (2019).

8. G. Nava-Arzaluz, M., Calderon-Lojero, I., Quintanar-Guerrero, D., Villalobos-Garcia, R. \& Ganem- 
Quintanar, a. Microneedles as Transdermal Delivery Systems: Combination with Other Enhancing Strategies. Curr. Drug Deliv. 9, 57-73 (2012).

9. Bal, S. M., Caussin, J., Pavel, S. \& Bouwstra, J. a. In vivo assessment of safety of microneedle arrays in human skin. Eur. J. Pharm. Sci. 35, 193-202 (2008).

10. Gill, S. E. \& Parks, W. C. Metalloproteinases and their inhibitors: Regulators of wound healing. Int. J. Biochem. Cell Biol. 40, 1334-1347 (2008).

11. Gupta, J., Gill, H. S., Andrews, S. N. \& Prausnitz, M. R. Kinetics of skin resealing after insertion of microneedles in human subjects. J. Control. Release 154, 148-155 (2011).

12. Han, T. Y. et al. Facial skin barrier function recovery after microneedle transdermal delivery treatment. Dermatologic Surg. 38, 1816-1822 (2012).

13. Ito, Y. et al. Dissolving Microneedles as Skin Allergy Test Device. Biol. Pharm. Bull. 40, 531-534 (2017).

14. Spina, L. et al. Comparison of microneedles and adhesive-tape stripping in skin preparation for epicutaneous allergen delivery. Int. Arch. Allergy Immunol. 167, 103-109 (2015).

15. Wiernsperger, N. et al. Microcirculation and Insulin Resistance. (2009). doi:10.2174/97816080505741090101

16. de Jongh, R. T., Sern??, E. H., IJzerman, R. G., J??rstad, H. T. \& Stehouwer, C. D. a. Impaired local microvascular vasodilatory effects of insulin and reduced skin microvascular vasomotion in obese women. Microvasc. Res. 75, 256-262 (2008).

17. Bal, S. M. et al. Influence of microneedle shape on the transport of a fluorescent dye into human skin in vivo. J. Control. Release 147, 218-224 (2010).

18. Medicine, E. Assessment of microvascular effects of vasoactive drugs Methodological in vivo studies in humans based on iontophoresis Joakim Henricson. (2009).

19. Haridass, I. N. et al. Cellular metabolism and pore lifetime of human skin following microprojection array mediation. J. Control. Release 306, 59-68 (2019).

20. Noh, Y. W. et al. In vitro characterization of the invasiveness of polymer microneedle against skin. Int. J. Pharm. 397, 201-205 (2010).

21. Haq, M. I. et al. Clinical administration of microneedles: Skin puncture, pain and sensation. Biomed. Microdevices 11, 35-47 (2009). 
Figure 1: Microneedle-induced effects in skin vasculature. (A) 1-hour time series: The RBC concentration (shown as $\mathrm{C}_{\mathrm{RBC}}$ ) was assessed using TIVI (Tissue Viability images) for 60 minutes, after applying different needle lengths $(300 \mu \mathrm{m}, 500 \mu \mathrm{m}, 750 \mu \mathrm{m})$ and different weights and durations (mean \pm SEM, $\mathrm{n}=8$ ).

(B) 48-hour time series. (C) Representative images of the 48-hour time series.

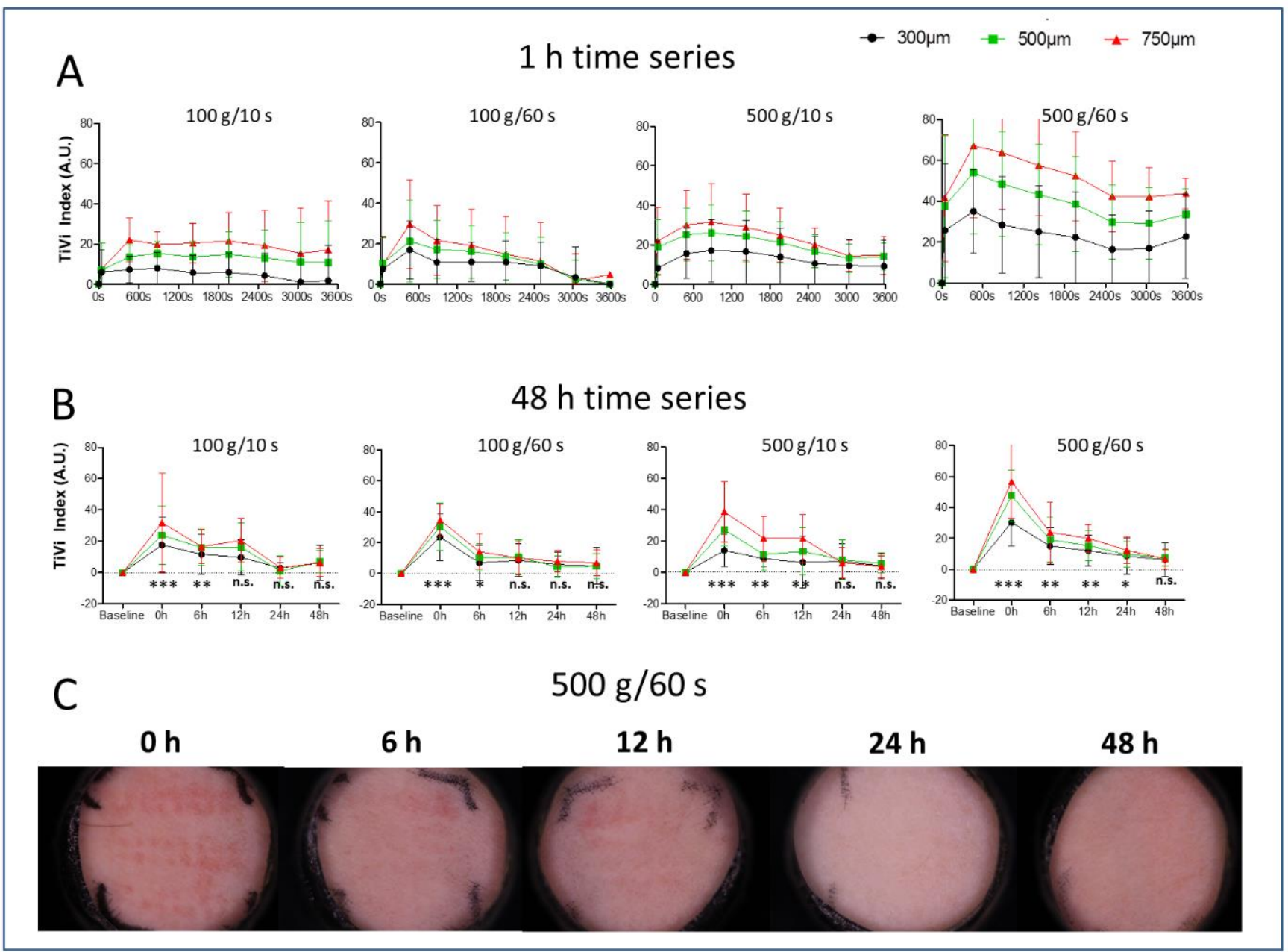


Table 1: Maximum $C_{R B C}$ for all different needle lengths and combinations of weight and duration ( $n=8$, mean $\pm S D$ ).

\begin{tabular}{|c|c|c|c|}
\hline \multicolumn{3}{|c|}{ Maximum responses for different needle lengths and } \\
combinations of weight and duration (n=8) \\
\hline times & $750 \mu \mathrm{m}$ & $500 \mu \mathrm{m}$ & $300 \mu \mathrm{m}$ \\
\hline $100 \mathrm{~g} / 10 \mathrm{~s}$ & $28.2 \pm 14.0$ & $21.7 \pm 9.0$ & $13.1 \pm 8.2^{* *}$ \\
\hline $100 \mathrm{~g} / 60 \mathrm{~s}$ & $28.7 \pm 17.3$ & $24.3 \pm 13.8$ & $20.7 \pm 14.5$ \\
\hline $500 \mathrm{~g} / 10 \mathrm{~s}$ & $38.4 \pm 18.7$ & $35.6 \pm 13.5$ & $25.6 \pm 19.9^{*}$ \\
\hline $500 \mathrm{~g} / 60 \mathrm{~s}$ & $81.8 \pm 29.0$ & $70.9 \pm 30.3$ & $50.7 \pm 31.1^{* * *}$ \\
\hline
\end{tabular}

Significant difference in $\mathrm{C}_{\mathrm{RBC}}$ increase compared to $750 \mu \mathrm{m}, * \mathrm{p}<0.05, * * p<0.01, * * * p<0.001$. 
Table 2: Significance of increase in $\mathrm{C}_{\mathrm{RBC}}$ for different durations and weights compared to baseline during the first 48 hours after microneedle application.

\begin{tabular}{|c|c|c|c|c|c|c|}
\hline \multicolumn{7}{|c|}{ Column stats analysis (2way ANOVA with multiple comparisons vs. Baseline [0], } \\
\hline Time[h] & length & $\max$ & $6 \mathrm{~h}$ & $12 \mathrm{~h}$ & $24 \mathrm{~h}$ & $48 \mathrm{~h}$ \\
\hline \multirow[t]{3}{*}{$100 \mathrm{~g} / 10 \mathrm{~s}$} & $750 \mu \mathrm{m}$ & $<0.001$ & n.s & $<0.001$ & n.s & n.s \\
\hline & $500 \mu \mathrm{m}$ & $<0.001$ & 0.005 & 0.004 & n.s & n.s \\
\hline & $300 \mu \mathrm{m}$ & 0.001 & n.s & n.s & n.s & n.s \\
\hline \multirow[t]{3}{*}{$100 \mathrm{~g} / 60 \mathrm{~s}$} & $750 \mu \mathrm{m}$ & $<0.001$ & 0.015 & n.s & n.s & n.s \\
\hline & $500 \mu \mathrm{m}$ & $<0.001$ & n.s & n.s & n.s & n.s \\
\hline & $300 \mu \mathrm{m}$ & $<0.001$ & n.s & n.s & n.s & n.s \\
\hline \multirow[t]{3}{*}{$500 \mathrm{~g} / 10 \mathrm{~s}$} & $750 \mu \mathrm{m}$ & $<0.001$ & $<0.001$ & $<0.001$ & n.s & n.s \\
\hline & $500 \mu \mathrm{m}$ & $<0.001$ & n.s & 0.03 & n.s & n.s \\
\hline & $300 \mu \mathrm{m}$ & 0.02 & n.s & n.s & n.s & n.s \\
\hline \multirow[t]{3}{*}{$500 \mathrm{~g} / 60 \mathrm{~s}$} & $750 \mu \mathrm{m}$ & $<0.001$ & $<0.001$ & $<0.001$ & 0.047 & n.s \\
\hline & $500 \mu \mathrm{m}$ & $<0.001$ & $<0.001$ & 0.008 & n.s & n.s \\
\hline & $300 \mu \mathrm{m}$ & $<0.001$ & 0.009 & n.s & n.s & n.s \\
\hline
\end{tabular}

